$T_{l}=T_{m}-2 \Delta_{0} \exp f\left(\Delta_{0}\right) \times \exp -l \sqrt{ } A$,

in view of which (6) may also be written in the convenient form,

$\Delta \exp f(\Delta)=\frac{1}{2}\left(T_{m}-T_{l}\right)(\exp q \sqrt{ } A+\exp -q \sqrt{ } A)$,

which is applicable over the whole length of a long filament.

National Physical Laboratory of India,

K. S. KRISHNAN

S. C. JAIN

Hillside Road,

New Delhi 12.

Feb. 12.

"See Carslaw, H. S., and Jaeger, J. C., "Conduction of Heat in Solids", 135 (Oxford : Clarendon Press, 1947).

${ }^{2}$ Krishnan, Sir K. S., and Jain, S. C., Nature, 173, 166 (1954) ; Proc.

Roy. Soc., A (in course of publication).

\section{A New Technique for investigating Surface Flow in Metal-working Processes}

DuRINa the plastic deformation of metals in shaping processes, there is relative movement between the flowing metal and the tools. An attempt was made by Siebel and Osenberg ${ }^{1}$ to deduce this relative movement by noting the distortion of a grid scribed to the first, and a curved scratch is produced. The final shape is like that of a fish-hook. Fig. 1 shows stages in the development of scratches through the arc of contact. The specimen was prepared by interrupting the rolling process and removing the strip for examination.

A preliminary investigation based on the measurement of surface scratches has led to several conclusions regarding the rolling process.

(1) The projected length of scratch in the rolling direction is close to the length predicted by a simple theory based on the assumption that the deformation is homogeneous.

(2) The neutral position, which is related to the frictional conditions in rolling, can be directly observed.

(3) The spread of elements in the surface can be measured by observation of the length of the scratch projected in the lateral direction.

(4) Since the direction of the growing end of a scratch within the region of contact coincides with the direction of the frictional force at that point, maps of frictional force direction in the region of contact may be constructed. In theories of rolling it has always been assumed that the frictional forces are parallel to the rolling direction. Observations have shown that even with small lateral spread (less than 1 per cent) this is far from being the case.

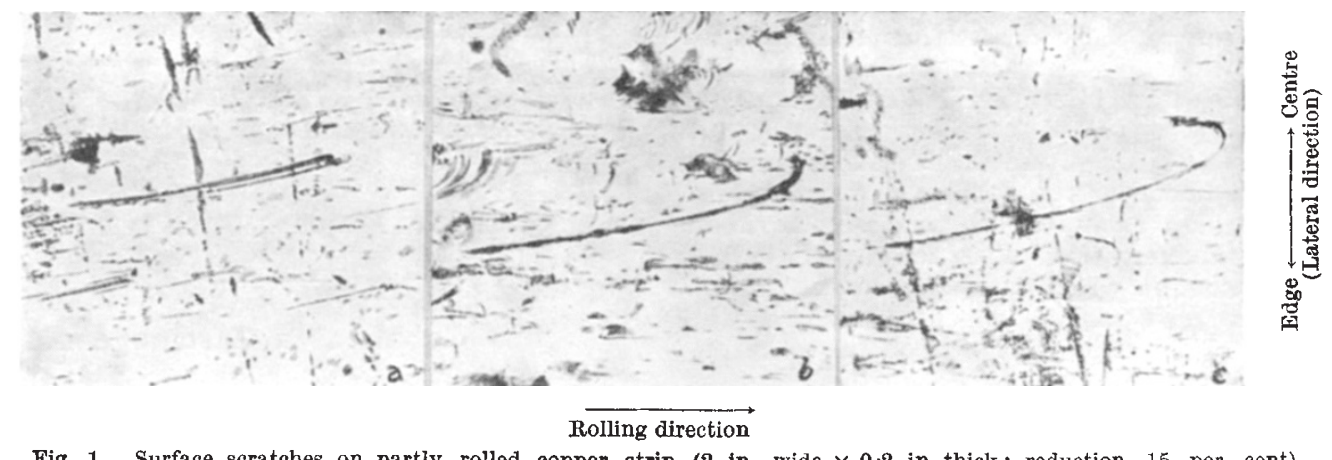

Fig. 1. Surface scratches on partly rolled copper strip (2 in. wide $\times 0.2$ in. thick: reduction, 15 per cent).

$$
(a) \text { Between entry and neutral position; }(b) \text { near neutral position; }(c) \text { at exit. } \times 150
$$

on the surface of strip before further rolling. The accuracy was not high, however, and the method yielded little information on surface flow.

It occurred to us that small projections on the surface of the rolls or tool faces might give rise to ploughed tracks on the surface of the metal undergoing deformation, and that such markings might provide illuminating evidence regarding the relative movement. We have observed that tracks or surface scratches are, in fact, produced by the adventitious minute projections normally present on rolls and platens. These surface scratches provide a valuable means of investigating the topography of surface flow during deformation.

When a metal strip is reduced in thickness by passing it between cylindrical rolls, its speed, on entering the zone of deformation, is less than that of the roll periphery. The strip.is accelerated until, at some 'neutral position' within the region of contact, roll and strip surfaces are moving with the same velocity, and the strip finally emerges moving more rapidly than the rolls. The relative movement which takes place generates the surface scratches mentioned above. Spreading in the lateral direction adds a second component of relative velocity at right angles
Further work using the technique described here is in progress.

J. M. CAPUS

M. G. COCKCROFT

Department of Industrial Metallurgy,

University of Birmingham.

Feb. 3.

${ }^{1}$ Siebel, E., and Osenberg, E., Mitt. K.-W.-Inst. f. Eisenforschung, 16, $33(1934)$.

\section{Refractive Index of Concentrated Protein Solutions}

AN accurate knowledge of the specific refraction increment $\alpha$, or $\mathrm{d} n / \mathrm{d} C$, of proteins is important in measurements which involve the use of the ultracentrifuge, electrophoresis and light-scattering. Recently, it has become important in connexion with cytological methods of refractometry ${ }^{1}$ and mass determination ${ }^{2}$ of living cells. The refraction increment has hitherto usually been measured only at rather low concentrations. The highest concentration reported seems to be by Howard ${ }^{3}$ in 1920, who found that the variation of refractive index with concentration of hæmoglobin was linear up to about 17 per 